\title{
An Enantioconvergent Benzylic Hydroxylation Using a Chiral Aryl lo- dide in a Dual Activation Mode
}

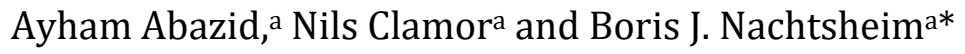 \\ University of Bremen, Institute of Organic and Analytical Chemistry, Leobener Straße 7, 28359 Bremen, Germany \\ nachtsheim@uni-bremen.de
}

KEYWORDS Chiral Alcohols, Enantioselective Oxidation, Chiral Alcohols, Enantioconvergent Catalysis, Hypervalent Iodine

\begin{abstract}
We report the application of a chiral triazole-substituted iodoarene in a direct enantioselective hydroxylation of alkyl arenes. This method allows the rapid synthesis of chiral benzyl alcohols in high yields and exceptional stereocontrol despite its non-templated nature. In a unique cascade activation comprising of an initial irradiation-induced radical $\mathrm{C}$ - $\mathrm{H}$ bromination and a consecutive enantioconvergent hydroxylation, the iodoarene catalyst has a dual role. It initiates the radical bromination in its oxidized state through an in situ formed bromoiodane and in the second, $\mathrm{Cu}$-catalyzed step, it acts as a chiral ligand. To the best of our knowledge this the first example demonstrating the ability of a chiral aryl iodide catalyst acting both, as an oxidant and as a chiral ligand in a highly enantioselective $\mathrm{C}$-H-activating transformation. Furthermore, this is the first example for an enantioconvergent hydroxylation.
\end{abstract}

\section{INTRODUCTION}

The direct hydroxylation of $\mathrm{C}\left(s p^{3}\right)-\mathrm{H}$ bonds is one of the most efficient reactions to introduce molecular complexity into unfunctionalized bulk chemicals. ${ }^{1,2}$ Once introduced, the resulting alcohols are either a fundamental part of the desired target molecule or they can be readily transformed into other useful functionalities. Hydroxylations of benzylic $\mathrm{C}\left(s p^{3}\right)-\mathrm{H}$ bonds are of particular interest due to the intrinsic reactivity of the benzylic $\mathrm{C}-\mathrm{H}$ bond allowing a highly regioselective $\mathrm{C}-\mathrm{H}$ activation in the presence of other aliphatic $\mathrm{C}-\mathrm{H}$ bonds. ${ }^{34}$ Based on cytochrome P-450 oxygenases as natural blueprint, ${ }^{5}$ a variety of chiral metal-porphyrine complexes were developed for enantioselective hydroxylations. ${ }^{6}$ In a first report in 1989 by Groves and co-workers chiral iron porphyrins could be applied in combination with iodosobenzene as oxygen-donor to hydroxylate ethyl benzene in $40 \%$ yield and $41 \%$ ee. ${ }^{7}$ Template-directed procedures give excellent selectivities although only well-selected substrates that can non-covalently interact with the chiral catalyst through hydrogen-bonding patterns can be addressed efficiently. ${ }^{8,9}$ It is surprising, that even more than three decades after the initial finding of Groves, simple alkyl benzenes are still difficult to hydroxylate in good yields and enantioselectivities through purely synthetic catalysts. ${ }^{10,11}$ Hypervalent iodine reagents are commonly used oxidants for oxidative coupling reagents and in group and oxygen transfer reactions. ${ }^{12,13,14}$ They can be also applied in benzylic hydroxylations as demonstrated by Groves in his early report as well as in high valent transition metal chemistry ${ }^{15}$ and in photoredox catalysis. ${ }^{16}$ The use of chiral iodanes or their aryl iodide precursors in catalytic enantioselective benzylic hydroxylations is so far unknown. ${ }^{17}$ During our systematic studies of $\mathrm{N}$-heterocycle-stabilized iodanes
(NHIs), ${ }^{18}$ we recently introduced triazole-substituted aryl iodides 1 (Figure 1) as chiral iodane-precursors and successfully verified their excellent performance in a plethora of enantioselective oxidative transformations. ${ }^{19}$ In a new approach we herein demonstrate their unique ability to act in a dual way, initially as a iodane-based halogen donor for a non-stereoselective radical-mediated halogenation and as a chiral ligand in a so far undescribed enantioconvergent hydroxylation. ${ }^{20,21}$

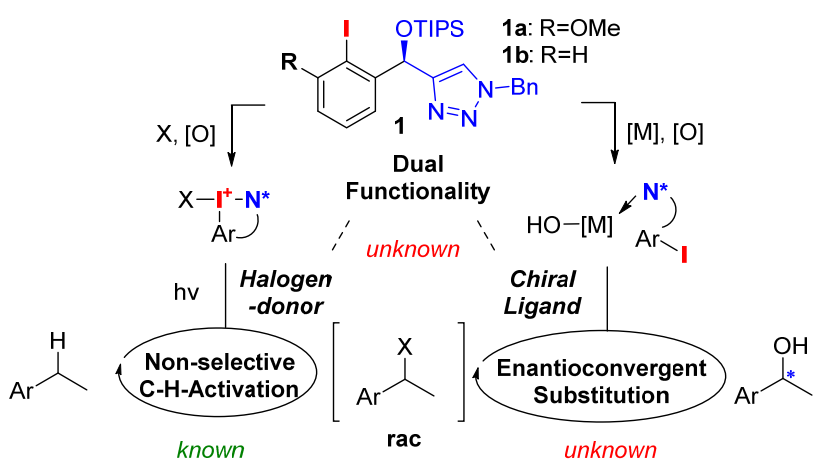

Figure 1. Working model for the dual activation of alkyl arenes using catalysts of type 1

Combining 1 with a co-oxidant and a halogen anionshould initially form a stabilized halogen(aryl)- $\lambda^{3}$-iodane, to initiate the $\mathrm{C}-\mathrm{H}$-activation and then ligate a transition metal during the second, enantioconvergent, reaction step. The combination of bromide sources and aryl- $\lambda^{3}$-iodanes is known to activate benzylic C-H-bonds through in situ formation of bromo-iodanes followed by a light-induced homolytic cleavage of the labile I-Br-bond. ${ }^{22}$ 


\section{RESULTS AND DISCUSSION}

Based on the fact that many radical-mediated enantioconvergent substitutions, in particular alkylations of secondary alkyl halides, can be catalyzed by $\mathrm{Cu}(\mathrm{I})$-salts, ${ }^{23,24} \mathrm{We}$ initially focused on $\mathrm{CuBr}$ acting as the bromide donor and the transition metal-catalyst. To test our hypothesis, the direct hydroxylation of ethyl benzene $\mathbf{2 a}$ was investigated (Table 1). As expected, under thermodynamic conditions in DCM no reaction was observed (entry 1 ). To our delight, irradiation with a blue LED gave the desired product $(\boldsymbol{R})$-3a in $68 \%$ yield and $71 \%$ ee using 150 mol\% CuBr (entry 2). While aromatic solvents such as toluene give a diminished yield and selectivity (entry 3) using acetonitrile gave $(\boldsymbol{R})$-3a in $75 \%$ yield and $90 \%$ ee (entry 4 ). Since the Cu-salt should initially only act as an activator for I-Br-bond activation, we were surprised by the observed high amounts that were needed for this additive and wondered whether it would be sufficient to add catalytic amounts of $\mathrm{Cu}(\mathrm{I}) \mathrm{Br}$ and another, cheaper, bromide source in stoichiometric quantities. Indeed, if only $20 \mathrm{~mol} \%$ of $\mathrm{CuBr}$ in combination with 1.5 equiv. of $\mathrm{NaBr}$ as additive were used, the reaction still proceeds with almost the same outcome (entry 5). Under these conditions we could even decrease catalyst loading of $\mathbf{1 a}$ to 15 mol\% without affecting yield and selectivity (entry 6). In agreement with our recent findings, catalyst $\mathbf{1 b}$ not bearing the important ortho-substituent in the aryl iodide, performs worse (see ESI, Scheme S1)

Table 1. Optimization of the reaction conditions.

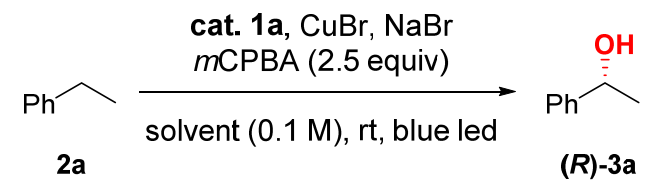

\begin{tabular}{|c|c|c|c|c|c|c|c|}
\hline $\begin{array}{c}\text { en- } \\
\text { try }\end{array}$ & $\mathbf{1 a}$ & $\mathbf{C u B r}$ & $\mathbf{N a B r}$ & $\begin{array}{c}\text { sol- } \\
\text { vent }\end{array}$ & $\begin{array}{c}\mathbf{t} \\
{[\mathrm{h}]}\end{array}$ & $\begin{array}{c}\text { yield } \\
{[\%]}\end{array}$ & $\begin{array}{c}\mathbf{e e} \\
{[\%]}\end{array}$ \\
\hline $1^{\mathrm{a}}$ & 20 & 150 & 0 & $\mathrm{DCM}$ & 12 & 0 & - \\
\hline 2 & 20 & 150 & 0 & $\mathrm{DCM}$ & 20 & 68 & 71 \\
\hline 3 & 20 & 150 & 0 & $\mathrm{PhMe}$ & 30 & 25 & 40 \\
\hline 4 & 20 & 150 & 0 & $\mathrm{CH}_{3} \mathrm{CN}$ & 14 & 75 & 90 \\
\hline 5 & 20 & 20 & 150 & $\mathrm{CH}_{3} \mathrm{CN}$ & 14 & 74 & 90 \\
\hline $\mathbf{6}^{\mathbf{b}}$ & $\mathbf{1 5}$ & $\mathbf{2 0}$ & $\mathbf{1 5 0}$ & $\mathrm{CH}_{3} \mathrm{CN}$ & $\mathbf{1 4}$ & $\mathbf{7 4}$ & $\mathbf{9 0}$ \\
\hline
\end{tabular}

aThe reaction was performed at $60^{\circ} \mathrm{C}$ for $12 \mathrm{hr}$. b These conditions will be further referred as "optimized conditions".

Under these optimized conditions, we examined the applicable substrate scope (Scheme 1). Initially, we investigated simple electron rich ethyl arenes. 4-Methyl- and 4methoxy-substituted derivatives reacted superior giving the desired benzyl alcohols $\mathbf{3 b}$ and $\mathbf{3} \mathbf{c}$ in high yields and $96 \%$ and $95 \%$ ee. Free hydroxy groups are tolerated as well giving 3d-3f in still good yields and 72\%-87\% ee.

We then examined the effect of electron withdrawing groups at the arene. All possible bromo-substituted isomers reacted well giving $\mathbf{3 g - 3 i}$ in throughout good yields of 76$79 \%$ with the best enantioselectivity being observed for the 3-Br-derivative (95\%). Ethyl-substituted nitro arenes gave compounds $\mathbf{3} \mathbf{j}$ and $\mathbf{3 k}$ in even better yields (90 and 89\%) and excellent $93 \%$ and $95 \%$ ee. Synthetically versatile nitriles and free carboxylic acids are also well tolerated yielding benzyl alcohols $\mathbf{3 l}$ and $\mathbf{3 m}$ in good yields and $83 \%$ and $95 \%$ ee. Indane and tetrahydronaphthalene give $\mathbf{3 n}$ and $\mathbf{3 o}$ in $79 \%$ and $77 \%$ yield with $85 \%$ and $95 \%$ ee. For these substrates, small amounts of dihydroxylation products $(<10 \%$ were observed). Next, the aliphatic side chain of the ethyl benzene was varied. $n$ Propyl- and $n$ butyl benzene gave $\mathbf{3 p}$ and $3 \mathbf{q}$ in yields and selectivities comparable to the model substrate. The same was observed for 1,2-diphenylethane giving $3 r$ in $79 \%$ and $93 \%$ ee. 2-Phenyl acetic yielded $(R)$ mandelic acid 3 s in $64 \%$ and $88 \%$ ee. We then examined multiple bond substitution in the benzylic side chain. Once again, the method showed a high robustness among those substrates. Allyl benzene was directly hydroxylated to yield the allyl alcohol 3t in 83\% ee. Propargyl alcohol 3u was isolated with even higher selectivities of $90 \%$ ee, although the yield was lower (61\%), probably due to a slight decomposition of the delicate propargylic alcohol. Finally, we investigated a variety of ethyl-substituted heterocycles as substrates.

\section{Scheme 1. Substrate Scope}

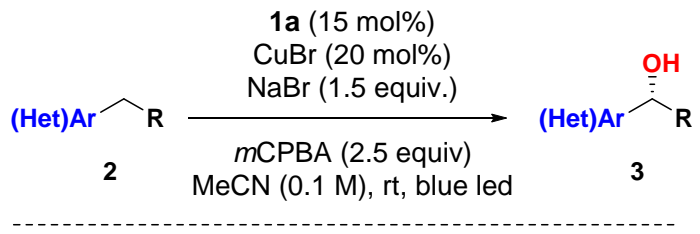

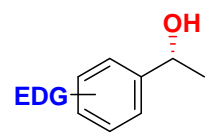

3b $(E D G=4-M e): 83 \%, 96 \%$ ee 3c (EDG = 4-OMe): $95 \%, 95 \%$ ee 3d (EDG = 2-OH): $58 \%, 84 \%$ ee 3e $(E D G=4-O H): 76 \%, 72 \%$ ee $3 f$ (EDG $=3-\mathrm{OH}): 68 \%, 87 \%$ ee<smiles>O[C@H]1C[N]c2ccccc21</smiles>

3n ( $n=1): 79 \%, 85 \%$ ee 3o $(n=2): 77 \%, 95 \%$ ee

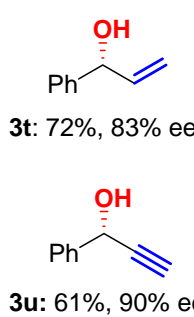

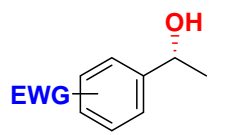

3 g $(E W G=4-B r): 76 \%, 87 \%$ ee 3h (EWG = 2-Br): $77 \%, 84 \%$ ee 3i (EWG = 3-Br): $79 \%, 95 \%$ ee 3j $\left(E W G=4-\mathrm{NO}_{2}\right): 90 \%, 93 \%$ ee 3k $\left(E W G=2-\mathrm{NO}_{2}\right): 89 \%, 95 \%$ ee 3I (EWG $=4-C N): 84 \%, 83 \%$ ee $3 \mathrm{~m}\left(\mathrm{EWG}=4-\mathrm{CO}_{2} \mathrm{H}\right): 74 \%, 95 \%$ ee

H $\quad 3 p(R=E t): 85 \%, 93 \%$ ee $3 q(\mathrm{R}=n \mathrm{Pr}): 77 \%, 85 \%$ ee $\mathrm{Ph}_{\mathbf{R}} \quad \begin{aligned} & 3 \mathbf{q}\left(\mathrm{R}=\mathrm{R}=\mathrm{CH}_{2} \mathrm{Ph}\right): 79 \%, 93 \% \text { ee } \\ & \mathbf{3}(\mathrm{R}): 77 \%, 85 \% \text { ee }\end{aligned}$ 3s: $64 \% 88 \%$ ee OH

$(\mathrm{X}=\mathrm{O})$ : no reaction 3v (X=S): 54\%, 89\% ee

3w (2-pyridyl): $63 \%, 78 \%$ ee 3x (4-pyridyl): $57 \%$, $87 \%$ ee<smiles>C[C@H](O)c1nc2ccccc2c(=O)[nH]1</smiles> 
While 2-ethyl furane did not react at all, the corresponding thiophene gave 3v in $89 \%$ ee and $54 \%$ yield. Even ethyl pyridines were tolerated under the applied oxidative conditions, despite potential oxygenations of the ring nitrogen. giving 2- and 4-pyridyl-substituted alcohols $\mathbf{3 w}$ and $\mathbf{3 x}$ in moderate yields and $78 \%$ and $87 \%$ ee. Even 4-ethyl quinazolinone could be directly hydroxylated to $3 \mathbf{y}$ with $80 \%$ ee.

\section{Scheme 2. Investigating the regioselectivity.}

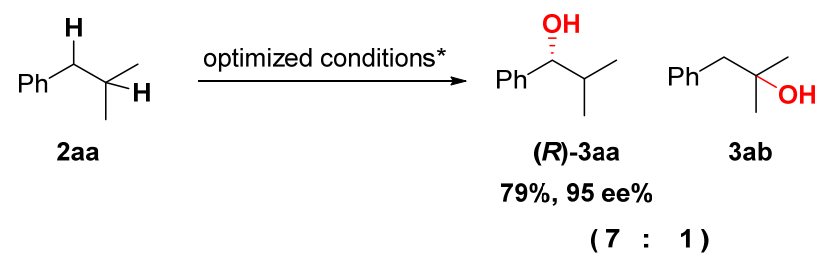

aAfter $4 \mathrm{~h}$ the reaction temperature was decreased to $0{ }^{\circ} \mathrm{C}$

We were also interested whether the applied reaction conditions allow the regioselective $\mathrm{C}$ - $\mathrm{H}$-activation of the benzyl C-H-bond in the presence of tertiary C-H-bonds - also potential hot spots for the initial radical-mediated halogenation. Therefore, we submitted substrate 2aa to our optimized reaction conditions and indeed found a mixture of regioisomers 3aa and 3ab in a ratio of 7:1 and 95\% ee for 3aa. (Scheme 2). Thus, the regioselective activation of the benzylic C-H is strongly favored.

After this comprehensive elaboration of the substrate scope we were intended to get a deeper understanding of the underlying reaction mechanism and in particular, proof the initially proposed dual role of the catalyst in this process and the unique enantioconvergent hydroxylation. For the first step, the radical-mediated halogenation, a brominated catalyst must be formed with a labile I-Br bond that easily breaks homolytic to induce the radical-mediated benzyl C$\mathrm{H}$-abstraction.

Scheme 3. Bromination with isolated catalyst $1 \mathrm{a}-\mathrm{Br}$ to prove the first step of the dual activation mechanism.

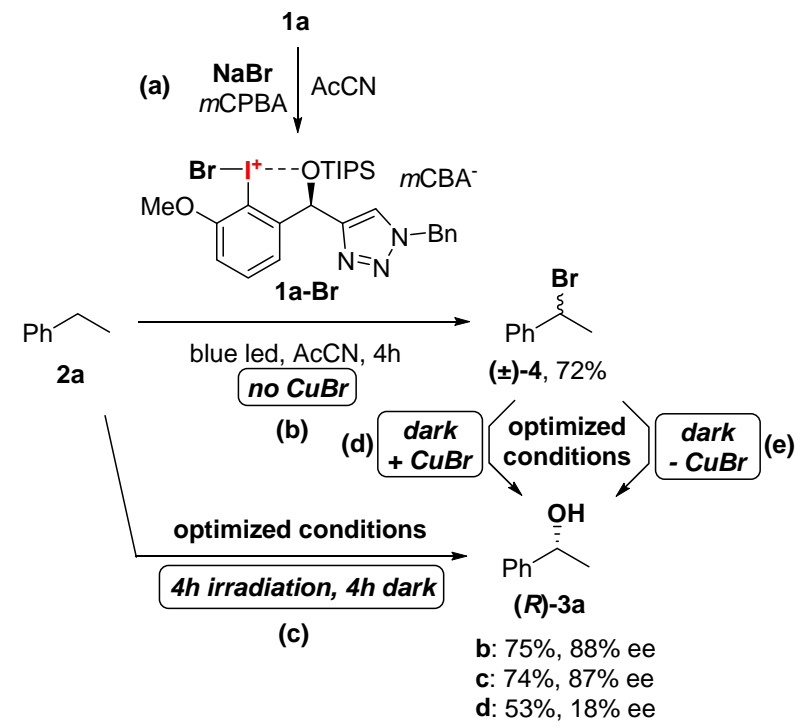

Treatment of 1 a with $\mathrm{NaBr}$ and $m \mathrm{CPBA}$ allowed us to isolate a white solid which we identified via NMR and MS as being the brominated derivative $\mathbf{1 a}-\mathbf{B r}$ (Scheme $3-\mathbf{a}$ ). Treatment of $2 \mathrm{a}$ with this compound without a $\mathrm{Cu}$-source under light irradiation yielded the benzyl bromide 4 in a very clean reaction and $72 \%$ yield as a racemate (b). Even if benzyl bromide would be formed enantioenriched its known tendency to racemize in polar solvents would yield the racemate under the given reaction conditions. ${ }^{25}$ This experiment implies, that once the benzyl bromide is formed, the second step of the transformation must be an enantioconvergent substitution in which irradiation should not be essential anymore. To verify this hypothesis, we reacted $2 \mathbf{a}$ again using the optimized conditions, but with only $4 \mathrm{~h}$ of light irradiation followed by further stirring for $4 \mathrm{~h}$ in the dark (c). Under these conditions the desired benzyl alcohol was observed in nearly the same yield and enantioselectivity as under fulltime irradiation. We then treated 4 under the optimized conditions, without irradiation (d) and isolated 3a in almost the same quantities and ee-values as in the previous experiments. We then questioned the necessity of $\mathrm{CuBr}$ as additive which was intended to catalyze exclusively the second step. When the reaction was repeated, without adding $\mathrm{CuBr}$ (e), 3a could still be isolated in 53\% yield, however, nearly as a racemate $(18 \%$ ee). From these experiments we conclude that $\mathrm{Cu}$ is not necessary for the initial $\mathrm{C}-\mathrm{H}$ activation but plays a fundamental role in the enantioconvergent hydroxylation. Here, 1a must act as a $N$-ligand coordinating through its triazole functionality, and therefore no oxidative conditions should be necessary. We therefore performed further control experiments starting from racemic $\mathbf{4}$ under our optimized conditions, without the addition of $m C$ PBA, but adding either $\mathrm{CuBr}$ or $\mathrm{CuBr}_{2}$ (Scheme $4-\mathrm{a}$ and $\mathrm{b}$ ). In both experiments no conversion to 3 a was observed. Thus, even in the second step the oxidant is necessary, but not just to oxidize the $\mathrm{Cu}(\mathrm{I})$-species. Obviously a hypervalent iodine compound must be important as well in the enantioconvergent step.

Scheme 4. Control experiments to elucidate the stereoconvergent substitution.

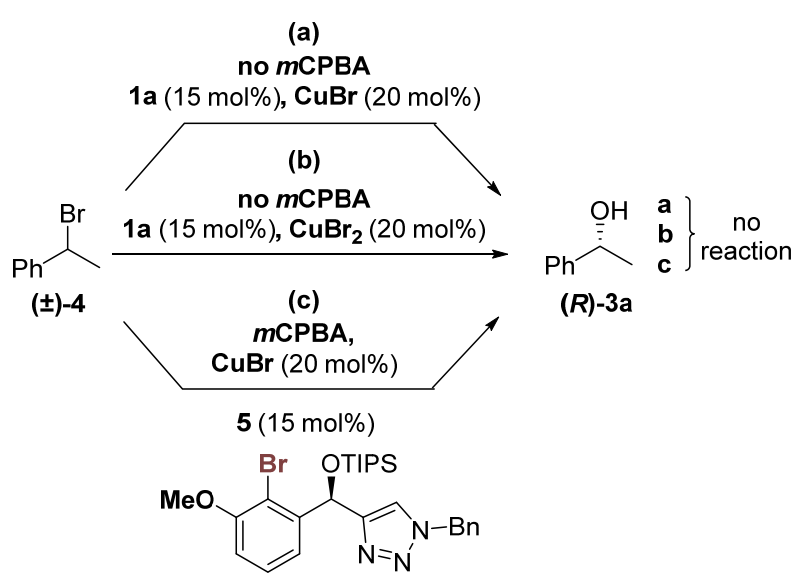

To proof this claim, we prepared catalyst derivative $\mathbf{5}$ bearing an aryl bromide instead of an aryl iodide. Aryl bromides cannot be oxidized with $m$ CPBA into a hypervalent state and therefore, as we observed, no reaction should occur (c). For gaining more insights about the potential active oxidation state of the involved $\mathrm{Cu}$-species, we repeated the 
experiment starting from benzyl bromide under oxidative conditions but now adding $\mathrm{CuBr}_{2}$ instead of $\mathrm{CuBr}$ (Scheme $5)$. Under these conditions 3a was isolated in slightly lower yields $(61 \%)$ but in similar enantioselectivity $(85 \%$ ee), again implying that the co-oxidant is necessary for the formation of a hydroxy iodane.

\section{Scheme 5. Enantioconvergent substitution with $\mathrm{CuBr}_{2}{ }^{\mathrm{a}}$}

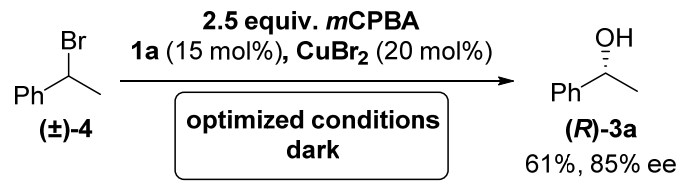

aReaction conditions: ( $\mathbf{\pm})-4(0.11 \mathrm{mmol}), \operatorname{MeCN}(0.1 \mathrm{M})$,

A hydroxylated catalyst 1a-0H could also not be isolated as a pure substrate for further control experiment due to its high reactivity based on the ortho-effect of the methoxy group and the induced hypervalent twist. ${ }^{26}$ However, NMR-experiments strongly support a hydroxylation under reductive collapse of the iodane as exemplified by $13 \mathrm{C}$ chemical shifts of the characteristic iodine-bound aryl signal (Figure 2). This ipso-carbon in 1a (blue) and the meta-carbon (green) have typical chemical shifts of approx. 90 and $110 \mathrm{ppm}(\mathrm{a})$. Upon addition of $m$ CPBA to yield $\mathbf{1 a - 0 H}$ as a mixture of different isomers, both signals clearly disappear and shift downfield to $>115$ ppm (b). Addition of $\mathrm{CuBr}$ regenerates $1 \mathrm{a}$ demonstrated by the reappearance of both signals (c). A significant line broadening of the signals in the corresponding proton NMR implies the existence of paramagnetic $\mathrm{Cu}(\mathrm{II})$ in the reaction mixture (see ESI). The transient formation of $\mathrm{Cu}(\mathrm{III})$-species cannot be ruled out and should be considered as well. Unfortunately, we were not able to isolate or characterize a $\mathrm{Cu}$-triazole complex therefore in depth understanding of the enantioconvergent step remains elusive.

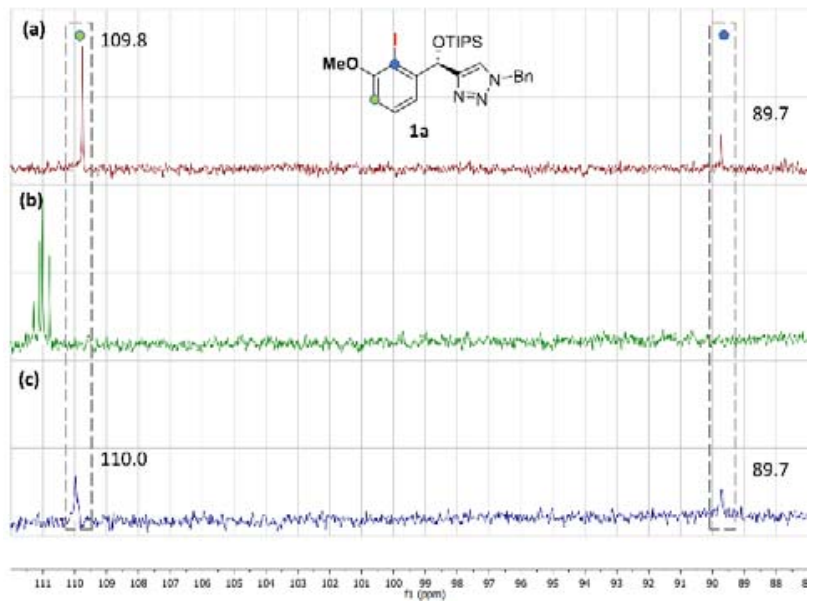

Figure 2. 13C-NMR-shifts for in situ oxidation of 1a between 85-112 ppm. (a) 1a alone. (b) 1a+mCPBA. (c) 1a + $m \mathrm{CPBA}+\mathrm{CuBr}$

However, based on these control experiments we want to propose a catalytic cycle shown in Scheme 6. In the initial irradiation-mediated cycle (Scheme 6 - a) 1a is first oxidized into the hydroxyiodobenzene $\mathbf{1 a - 0 H}$. Ligand exchange with $\mathrm{NaBr}$ gives the bromoiodobenze $\mathbf{1 a - B r}$ which underlies rapid I-Br bond cleavage under irradiation yielding a bromine radical and the radical cation 1a-RC. Radical benzylic bromination initiated by either of both species converts the alkyl benzene into the benzyl bromide as a racemate. In the second cycle (Scheme 6 - b) 1a is oxidized again in the initial-step to 1a-OH. The iodane then hydroxylates the Cu-salt either yielding a chiral $\mathrm{Cu}(\mathrm{II})$ or a $\mathrm{Cu}(\mathrm{III})-$ hydroxy complex 1a-Cu-OH.

\section{Scheme 6. Mechanistic proposal}

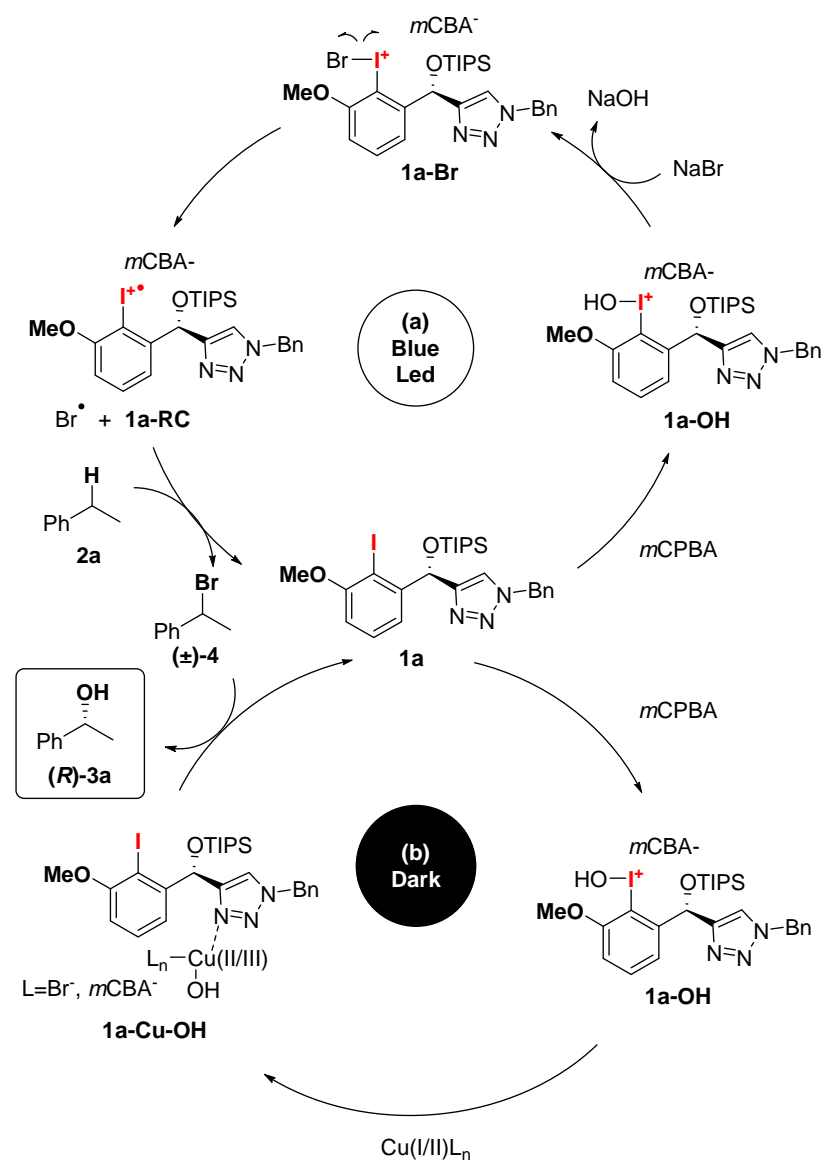

Depending on the oxidation state of the active $\mathrm{Cu}$-complex, either a radical or a $S_{N}$-type enantioconvergent substitution with the emerging benzyl bromide from cycle (a) yields $(\boldsymbol{R})$-3a. Due to the high racemization tendency of $\mathbf{4}$ we suggest this overall reaction to be a stereomutative enantioconvergent reaction with significantly different reaction rates for both enantiomers.

\section{CONCLUSIONS}

In conclusion this work demonstrates the ability of chiral aryl iodides substituted with $N$-heterocycles to be used in enantioselective benzylic hydroxylations through a double role, acting as oxidant and as a chiral ligand in a consecutive reaction sequence consisting of a benzyl bromination and a $\mathrm{Cu}$-mediated substitution. The verification of $( \pm)$-benzyl bromide as a reaction intermediate verifies that the second, $\mathrm{Cu}$-mediated reaction, is a unique enantioconvergent hydroxylation. The reaction tolerates a wide variety of substrates including electron rich and electron poor alkyl benzenes, exocyclic $\pi$-bonds as well as alkyl-substituted $N$-heterocycles. Furthermore, a high regioselectivity for the ben- 
zylic $\mathrm{C}$-H-bond in the presence tertiary $\mathrm{C}$-H-bonds was observed. This unique reaction cascade offers great potential for other enantioselective oxidative benzylic $\mathrm{C}$-H-activations, although the mechanism of the enantioconvergent step still needs to be further elaborated.

\section{ASSOCIATED CONTENT}

Supporting Information. The Supporting Information is available free of charge on the ACS Publications website at DOI: XXX. Including all experimental procedures and characterization data.

\section{AUTHOR INFORMATION}

\section{Corresponding Author}

*nachtsheim@uni-bremen.de

\section{Author Contributions}

The manuscript was written by BJN. All authors have given approval to the final version of the manuscript. / $¥$ These authors contributed equally. (match statement to author names with a symbol)

\section{Orcid}

Boris J. Nachtsheim: 0000-0002-3759-2770

\section{Funding Sources}

This work is support by the DFG (Deutsche Forschungsgemeinschaft - Grant NA 955/3-1).

Notes

The authors declare no competing financial interests.

\section{ACKNOWLEDGMENT}

Ayham Abazid acknowledges the State of Bremen for a Scholars at Risk Fund.

\section{ABBREVIATIONS}

$m$ CPBA: meta-Chloroperbenzoic acid; mCBA: meta-Chlorobenzoic acid; DCM: Dichloromethane

\section{REFERENCES}

(1) (a) Bryliakov, K. P. Catalytic Asymmetric Oxygenations with the Environmentally Benign Oxidants $\mathrm{H}_{2} \mathrm{O}_{2}$ and $\mathrm{O}_{2}$. Chem. Rev. 2017, 117, 11406. (b) Cernak, T.; Dykstra, K. D.; Tyagarajan, S.; Vachal, P.; Krska, S. W. The Medicinal Chemist's Toolbox for Late Stage Functionalization of Drug-like Molecules. Chem. Soc. Rev. 2016, 45, 546.

(2) White, M. C.; Zhao, J. Aliphatic C-H Oxidations for Late-Stage Functionalization. J. Am. Chem. Soc. 2018, 140, 13988.

(3) Genovino, J.; Sames, D.; Hamann, L. G.; Touré, B. B. Accessing Drug Metabolites via Transition-Metal Catalyzed C-H Oxidation: The Liver as Synthetic Inspiration. Angew. Chem. Int. Ed. 2016, 55, 14218.

(4) (a) Das, A.; Nutting, J. E.; Stahl, S. S. Electrochemical C-H Oxygenation and Alcohol Dehydrogenation involving Fe-oxo Species using Water as the Oxygen Source. Chem. Sci. 2019, 10, 7542. (b) Tanwar, L.; Börgel, J.; Ritter, T. Synthesis of Benzylic Alcohols by CH Oxidation. J. Am. Chem. Soc. 2019, 141, 17983.

(5) (a) Fasan, R. Tuning P450 Enzymes as Oxidation Catalysts. ACS Catal. 2012, 2, 647. (b) Denisov, I. G.; Makris, T. M.; Sligar, S. G.; Schlichting, I. Structure and Chemistry of Cytochrome P450. Chem. Rev. 2005, 105, 2253.
(6) (a) Guo, M.; Corona, T.; Ray, K.; Nam, W. Heme and Nonheme High-Valent Iron and Manganese Oxo Cores in Biological and Abiological Oxidation Reactions. ACS Cent. Sci. 2019, 5, 13. (b) Petsi, M.; Zografos, A. L. Advances in Catalytic Aerobic Oxidations by Activation of Dioxygen-Monooxygenase Enzymes and Biomimetics. Synthesis 2018, 50, 4715. (c) Adam, S. M.; Wijeratne, G. B.; Rogler, P. J.; Diaz, D. E.; Quist, D. A.; Liu, J. J.; Karlin, K. D. Synthetic Fe/Cu Complexes: Toward Understanding Heme-Copper Oxidase Structure and Function. Chem. Rev. 2018, 118, 10840.

(7) Groves, J. T.; Viski, P. Asymmetric Hydroxylation by a Chiral Iron Porphyrin. J. Am. Chem. Soc. 1989, 111, 8537.

(8) Carboni, S.; Gennari, C.; Pignataro, L.; Piarulli, U. Supramolecular Ligand-Ligand and Ligand-Substrate Interactions for Highly Selective Transition Metal Catalysis. Dalton Trans 2011, 40, 4355.

(9) (a) Burg, F.; Breitenlechner, S.; Jandl, C.; Bach, T. Enantioselective Oxygenation of Exocyclic Methylene Groups by a Manganese Porphyrin Catalyst with a Chiral Recognition Site. Chem. Sci. 2020, 11, 2121. (b) Burg, F.; Bach, T. Lactam Hydrogen Bonds as Control Elements in Enantioselective Transition-Metal-Catalyzed and Photochemical Reactions. J. Org. Chem. 2019, 84, 8815. (c) Burg, F.; Gicquel, M.; Breitenlechner, S.; Pothig, A.; Bach, T. Site- and Enantioselective C-H Oxygenation Catalyzed by a Chiral Manganese Porphyrin Complex with a Remote Binding Site. Angew. Chem. Int. Ed. 2018, 57, 2953. (d) Frost, J. R.; Huber, S. M.; Breitenlechner, S.; Bannwarth, C.; Bach, T. Enantiotopos-Selective C-H Oxygenation Catalyzed by a Supramolecular Ruthenium Complex. Angew. Chem. Int. Ed. 2015, 54, 691.

(10) Hartwig, J. F.; Larsen, M. A. Undirected, Homogeneous C-H Bond Functionalization: Challenges and Opportunities. ACS Cent. Sci. 2016, 2, 281.

(11) (a) Betori, R. C.; May, C. M.; Scheidt, K. A. Combined Photoredox/Enzymatic C-H Benzylic Hydroxylations. Angew. Chem. Int. Ed. 2019, 58, 16490. (b) Ottenbacher, R. V.; Talsi, E. P.; Rybalova, T. V.; Bryliakov, K. P. Enantioselective Benzylic Hydroxylation of Arylalkanes with $\mathrm{H}_{2} \mathrm{O}_{2}$ in Fluorinated Alcohols in the Presence of Chiral Mn Aminopyridine Complexes. ChemCatChem 2018, 10, 5323. (c) Talsi, E. P.; Samsonenko, D. G.; Ottenbacher, R. V.; Bryliakov, K. P. Highly Enantioselective C-H Oxidation of Arylalkanes with $\mathrm{H} 2 \mathrm{O} 2$ in the Presence of Chiral Mn-Aminopyridine Complexes. ChemCatChem 2017, 9, 4580. (d) Gross, Z.; Ini, S. Asymmetric Catalysis by a Chiral Ruthenium Porphyrin: Epoxidation, Hydroxylation, and Partial Kinetic Resolution of Hydrocarbons. Org. Lett. 1999, 1, 2077. (e) Hamada, T.; Irie, R.; Mihara, J.; Hamachi, K.; Katsuki, T. Highly Enantioselective Benzylic Hydroxylation with Concave Type of (Salen)manganese(III) Complex. Tetrahedron 1998, 54, 10017. (f) Hamachi, K.; Irie, R.; Katsuki, T. Asymmetric Benzylic Oxidation Using a Mn-salen Complex as Catalyst. Tetrahedron Lett. 1996, 37, 4979.

(12) Hypervalent Iodine Chemistry; Wirth, T., Ed.; Top. Curr. Chem. 373; Springer: Cham, 2016.

(13) Zhdankin, V. V. Hypervalent Iodine Chemistry: Preparation, Structure and Synthetic Applications of Polyvalent Iodine Compounds; John Wiley \& Sons Inc: Chichester, West Sussex, 2014.

(14) (a) Hyatt, I. F. D.; Dave, L.; David, N.; Kaur, K.; Medard, M.; Mowdawalla, C. Hypervalent Iodine Reactions Utilized in 'CarbonCarbon Bond Formations. Org. Biomol. Chem. 2019, 17, 7822. (b) Budhwan, R.; Yadav, S.; Murarka, S. Late Stage Functionalization of Heterocycles using Hypervalent Iodine(III) Reagents. Org. Biomol. Chem. 2019, 17, 6326. (c) Lee; Choi; Hong. Alkene Difunctionalization Using Hypervalent Iodine Reagents: Progress and Developments in the Past Ten Years. Molecules 2019, 24, 2634. (d) Reddy Kandimalla, S.; Prathima Parvathaneni, S.; Sabitha, G.; Subba Reddy, B. V. Recent Advances in Intramolecular Metal-Free Oxidative $\mathrm{C}-\mathrm{H}$ Bond Aminations Using Hypervalent Iodine(III) Reagents. Eur. J. Org. Chem. 2019, 2019, 1687. (e) Xing, L.; Zhang, Y.; Du, Y. Hypervalent Iodine-Mediated Synthesis of Spiroheterocycles via Oxidative Cyclization. Curr. Org. Chem. 2019, 23, 14. (f) Ghosh, M. 
K.; Rajkiewicz, A. A.; Kalek, M. Organocatalytic Group Transfer Reactions with Hypervalent Iodine Reagents. Synthesis 2019, 51, 359. (g) Hari, D. P.; Caramenti, P.; Waser, J. Cyclic Hypervalent Iodine Reagents: Enabling Tools for Bond Disconnection via Reactivity Umpolung. Acc. Chem. Res. 2018, 51, 3212. (h) Elsherbini, M.; Wirth, T. Hypervalent Iodine Reagents by Anodic Oxidation: A Powerful Green Synthesis. Chem. Eur. J. 2018, 24, 13399. (i) Boelke, A.; Finkbeiner, P.; Nachtsheim, B. J. Atom-Economical Group-Transfer Reactions with Hypervalent Iodine Compounds. Beilstein J. Org. Chem. 2018, 14, 1263. (j) Yoshimura, A.; Yusubov, M. S.; Zhdankin, V. V. Synthetic Applications of Pseudocyclic Hypervalent Iodine Compounds. Org. Biomol. Chem. 2016, 14, 4771. (k) Li, Y.; Hari, D. P.; Vita, M. V.; Waser, J. Cyclic Hypervalent Iodine Reagents for Atom-Transfer Reactions: Beyond Trifluoromethylation. Angew. Chem. Int. Ed. 2016, 55, 4436. (l) Yoshimura, A.; Zhdankin, V. V. Advances in Synthetic Applications of Hypervalent Iodine Compounds. Chem. Rev. 2016, 116, 3328.

(15) Sousa e Silva, F. C.; Tierno, A. F.; Wengryniuk, S. E. Hypervalent Iodine Reagents in High Valent Transition Metal Chemistry. Molecules 2017, 22, DOI: 10.3390 /molecules 22050780 .

(16) Wang, L.; Liu, J. Synthetic Applications of Hypervalent Iodine(III) Reagents Enabled by Visible Light Photoredox Catalysis. Eur. J. Org. Chem. 2016, 2016, 1813.

(17) (a) Parra, A. Chiral Hypervalent Iodines: Active Players in Asymmetric Synthesis. Chem. Rev. 2019, 119, 12033. (b) Flores, A.; Cots, E.; Bergès, J.; Muñiz, K. Enantioselective Iodine(I/III) Catalysis in Organic Synthesis. Adv. Synth. Catal. 2019, 361, 2. (c) Ghosh, S.; Pradhan, S.; Chatterjee, I. A Survey of Chiral Hypervalent Iodine Reagents in Asymmetric Synthesis. Beilstein J. Org. Chem. 2018, 14, 1244. (d) Claraz, A.; Masson, G. Asymmetric Iodine Catalysis-mediated Enantioselective Oxidative Transformations. Org. Biomol. Chem. 2018, 16, 5386.

(18) (a) Boelke, A.; Nachtsheim, B. J. Evolution of N-HeterocycleSubstituted Iodoarenes (NHIAs) to Efficient Organocatalysts in Iodine(I/III)-Mediated Oxidative Transformations. Adv. Synth. Catal. 2020, 362, 184. (b) Boelke, A.; Vlasenko, Y. A.; Yusubov, M. S.; Nachtsheim, B. J.; Postnikov, P. S. Thermal Stability of N-Heterocycle-Stabilized Iodanes - A Systematic Investigation. Beilstein J. Org. Chem. 2019, 15, 2311. (c) Boelke, A.; Lork, E.; Nachtsheim, B. J. NHeterocycle-Stabilized Iodanes: From Structure to Reactivity. Chem. Eur. J. 2018, 24, 18653.

(19) (a) Abazid, A. H.; Nachtsheim, B. J. A Triazole-Substituted Aryl Iodide with Omnipotent Reactivity in Enantioselective Oxidations. Angew. Chem. Int. Ed. 2020, 59, 1479. (b) Hempel, C.; MaichleMössmer, C.; Pericàs, M. A.; Nachtsheim, B. J. Modular Synthesis of Triazole-Based Chiral Iodoarenes for Enantioselective Spirocyclizations. Adv. Synth. Catal. 2017, 359, 2931.

(20) (a) Bhat, V.; Welin, E. R.; Guo, X.; Stoltz, B. M. Advances in Stereoconvergent Catalysis from 2005 to 2015: Transition-Metal-Mediated Stereoablative Reactions, Dynamic Kinetic Resolutions, and Dynamic Kinetic Asymmetric Transformations. Chem. Rev. 2017, 117, 4528. (b) Bartlett, S. L.; Johnson, J. S. Synthesis of Complex Glycolates by Enantioconvergent Addition Reactions. Acc. Chem. Res. 2017, 50, 2284. (c) Mohr, J. T.; Moore, J. T.; Stoltz, B. M. Enantioconvergent Catalysis. Beilstein J. Org. Chem. 2016, 12, 2038.

(21) (a) Chang, C.-H.; Sathishkumar, N.; Liao, Y.-T.; Chen, H.-T.; Han, J.-L. Solvent-Dependent Enantiodivergent Friedel-Crafts Reaction of Arylsulfonyl Indoles with 1-Naphthols. Adv. Synth. Catal. 2020, 362, 903. (b) Huo, H.; Gorsline, B. J.; Fu, G. C. Catalyst-controlled Doubly Enantioconvergent Coupling of Racemic Alkyl Nucleophiles and Electrophiles. Science 2020, 367, 559. (c) Zeng, G.; Li, Y.; Qiao, B.; Zhao, X.; Jiang, Z. Photoredox Asymmetric Catalytic Enantioconvergent Substitution of 3-Chlorooxindoles. Chem. Commun. 2019 55, 11362. (d) Schwarzwalder, G. M.; Matier, C. D.; Fu, G. C. Enantioconvergent Cross-Couplings of Alkyl Electrophiles: The Catalytic Asymmetric Synthesis of Organosilanes. Angew. Chem. Int. Ed. 2019, 58, 3571. (e) Iwamoto, T.; Okuzono, C.; Adak, L.; Jin, M.; Nakamura, M. Iron-Catalysed Enantioselective Suzuki-Miyaura Coupling of Racemic Alkyl Bromides. Chem. Commun. 2019, 55, 1128. (f) Zhang, X.; Ren, J.; Tan, S. M.; Tan, D.; Lee, R.; Tan, C.-H. An Enantioconvergent Halogenophilic Nucleophilic Substitution (SN2X) Reaction. Science 2019, 363, 400. (g) Wendlandt, A. E.; Vangal, P.; Jacobsen, E. N. Quaternary stereocentres via an enantioconvergent catalytic $\mathrm{S}_{\mathrm{N}} 1$ reaction. Nature 2018, 556, 447. (h) Mixdorf, J. C.; Sorlin, A. M.; Zhang, Q.; Nguyen, H. M. Asymmetric Synthesis of Allylic Fluorides via Fluorination of Racemic Allylic Trichloroacetimidates Catalyzed by a Chiral Diene-Iridium Complex. ACS Catal. 2018, 8 , 790. (i) Schäfer, P.; Sidera, M.; Palacin, T.; Fletcher, S. P. Asymmetric Cross-Coupling of Alkyl, Alkenyl and (Hetero)aryl Nucleophiles with Racemic Allyl Halides. Chem. Commun. 2017, 53, 12499.

(22) (a) Dohi, T.; Takenaga, N.; Goto, A.; Fujioka, H.; Kita, Y. Clean and Efficient Benzylic C-H Oxidation in Water Using a Hypervalent Iodine Reagent: Activation of Polymeric Iodosobenzene with $\mathrm{KBr}$ in the Presence of Montmorillonite-K10. J. Org. Chem. 2008, 73, 7365. (b) Tohma, H.; Maegawa, T.; Takizawa, S.; Kita, Y. Facile and Clean Oxidation of Alcohols in Water Using Hypervalent Iodine(III) Reagents. Adv. Synth. Catal. 2002, 344, 328. (c) Amey, R. L.; Martin, J. C. Synthesis and Reaction of Substituted Arylalkoxyiodinanes: Formation of stable bromoarylalkoxy and aryldialkoxy heterocyclic derivatives of tricoordinate organoiodine(III). J. Org. Chem. 1979, 44, 1779 .

(23) Gu, Q.-S.; Li, Z.-L.; Liu, X.-Y. Copper(I)-Catalyzed Asymmetric Reactions Involving Radicals. Acc. Chem. Res. 2020, 53, 170.

(24) (a) Bartoszewicz, A.; Matier, C. D.; Fu, G. C. Enantioconvergent Alkylations of Amines by Alkyl Electrophiles: Copper-Catalyzed Nucleophilic Substitutions of Racemic $\alpha$-Halolactams by Indoles. J. Am. Chem. Soc. 2019, 141, 14864. (b) Kainz, Q. M.; Matier, C. D.; Bartoszewicz, A.; Zultanski, S. L.; Peters, J. C.; Fu, G. C. Asymmetric copper-catalyzed $\mathrm{C}-\mathrm{N}$ cross-couplings induced by visible light. Science 16, 351, 681. (c) Ratani, T. S.; Bachman, S.; Fu, G. C.; Peters, J. C. Photoinduced, Copper-Catalyzed Carbon-Carbon Bond Formation with Alkyl Electrophiles: Cyanation of Unactivated Secondary Alkyl Chlorides at Room Temperature. J. Am. Chem. Soc. 2015, 137, 13902. (d) Do, H.-Q.; Bachman, S.; Bissember, A. C.; Peters, J. C.; Fu, G. C. Photoinduced, Copper-Catalyzed Alkylation of Amides with Unactivated Secondary Alkyl Halides at Room Temperature. J. Am. Chem. Soc. 2014, 136, 2162.

(25) Li, C.; Zhang, Y.; Sun, Q.; Gu, T.; Peng, H.; Tang, W. TransitionMetal-Free Stereospecific Cross-Coupling with Alkenylboronic Acids as Nucleophiles. J. Am. Chem. Soc. 2016, 138, 10774.

(26) (a) Thérien, M.-È.; Guilbault, A.-A.; Legault, C. Y. New Chiral Iodooxazoline Catalysts for the I(III)-Mediated $\alpha$-Tosyloxylation of Ketones: Refining the stereoinduction model. Tetrahedron: Asymmetry 2013, 24, 1193. (b) Guilbault, A.-A.; Legault, C. Y. Drastic Enhancement of Activity in Iodane-Based $\alpha$-Tosyloxylation of Ketones: Iodine(III) Does the Hypervalent Twist. ACS Catal. 2012, 2, 219. 


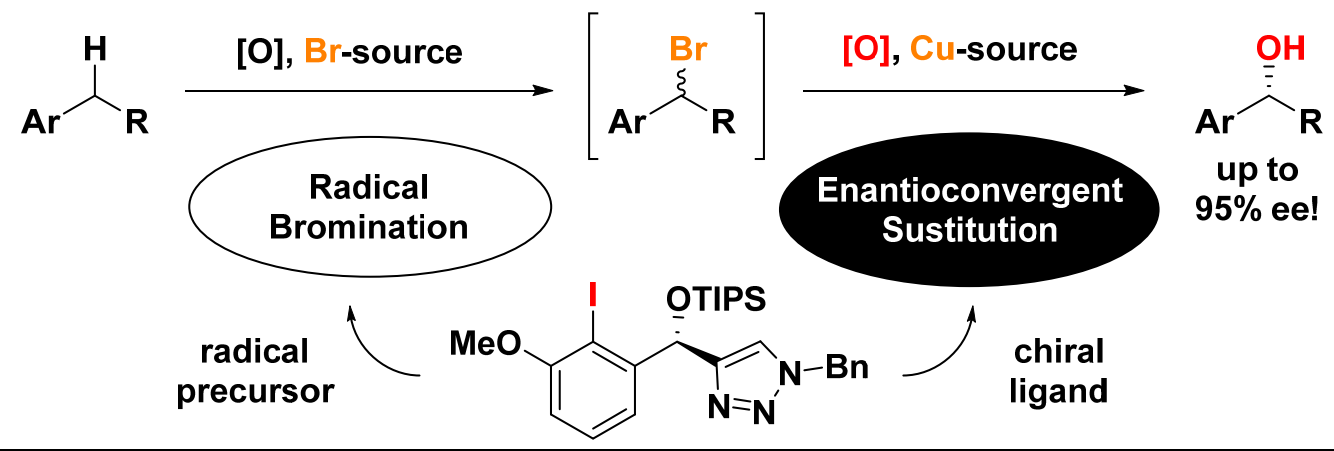

